\title{
CAPÍTULO 58 \\ INOVAÇÃO EM CIRURGIAS CRANIOFACIAIS, VANTAGENS E \\ INDICAÇÕES DO PLANEJAMENTO CIRÚRGICO \\ TRIDIMENSIONAL: UMA REVISÃO INTEGRATIVA
}

$\underline{\text { DOI 10.4322/978-65-995353-2-1.c58 }}$

\begin{abstract}
Bruno Romano de Oliveira ${ }^{1}$, Laryssa Costa Huguenin França ${ }^{2}$, Ana Paula Silva Carvalho $^{3}$, Robert Wilson da Silva Tostes ${ }^{4}$, Paula Mylena Paiva de Souza ${ }^{5}$, Weslley da Silva de Paiva ${ }^{6}$, Nyali Rosa de Castro ${ }^{7}$, Karla Arrigoni Gomes ${ }^{8}$, Maria Luíza da Costa
\end{abstract}

Gomes $^{9}$, Eduardo Stehling Urbano ${ }^{10}$

1 Faculdade de Odontologia/ Universidade Federal de Juiz de Fora, (bruno.romano12@gmail.com)

2 Faculdade de Odontologia/ Universidade Federal de Juiz de Fora,

(laryssa.huguenin@odontologia.ufjf.br)

3 Faculdade de Odontologia/ Universidade Federal de Juiz de Fora,

(anapaula.carvalho@estudante.ufjf.br)

4 Faculdade de Odontologia/ Universidade Federal de Juiz de Fora, (robert.tostes@odontologia.ufjf.br)

5 Faculdade de Odontologia/ Universidade Federal de Juiz de Fora,

(paula.mylena@odontologia.ufjf.br)

6 Faculdade de Odontologia/ Universidade Federal de Juiz de Fora,

(weslley.paiva@odontologia.ufjf.br)

7 Faculdade de Odontologia/ Universidade Federal de Juiz de Fora, (nyali.castro@odontologia.ufjf.br)

8 Faculdade de Odontologia/ Universidade Federal de Juiz de Fora, (karla.arrigoni@gmail.com)

9 Faculdade de Odontologia/ Universidade Federal de Juiz de Fora, (marialuiza.gomes@estudante.ufjf.br) 10 Instituto de Ciências Biológicas/Universidade Federal de Juiz de Fora,

\section{Resumo}

(esurss@yahoo.com.br)

Objetivo: Analisar as vantagens e indicações do planejamento cirúrgico virtual, destacando as inovações geradas por esse advento para a prática cirúrgica. Método: Foram incluídos estudos observacionais, revisões sistemáticas e revisões integrativas publicadas em inglês e/ou português entre janeiro de 2015 a dezembro de 2021. Foram excluídos duplicatas, cartas, editoriais, resumos de conferências, estudos em animais, e relato de casos com $\geq 5$ pacientes. As bases de dados utilizadas foram Medline (via Pubmed) e Biblioteca Virtual em Saúde (BVS). A base de dados Pubmed gerou 31 artigos, e a BVS 33 dos quais foram excluídos na primeira etapa respectivamente 8 e 32 artigos. Na segunda etapa, dos 24 artigos selecionados, mais 6 foram excluídos, e, após toda a análise, restaram 18 artigos incluídos no estudo. Resultados: O planejamento tridimensional apresenta inúmeras vantagens, como simulação 
virtual de osteotomias, confecção de guias cirúrgicos, melhor visualização das estruturas ósseas, o que garante um resultado mais satisfatório. Conclusão: O planejamento tridimensional representa uma inovação em cirurgia bucomaxilofacial. Este apresenta vantagens sobre o planejamento bidimensional, pois permite ao cirurgião realizar a avaliação do paciente com informações e imagens de estruturas craniofaciais livres de distorção de perspectiva, o que garante um melhor tratamento de deformidades, principalmente nas assimetrias unilaterais. No planejamento do tratamento há reduções de etapas e a utilização da técnica CAD / CAM permite uma maior precisão e previsibilidade, obtendo assim, resultados mais satisfatórios.

Descritores: Cirurgia Ortognática; Planejamento Cirúrgico; Planejamento Cirúrgico Virtual Área Temática: Ciências da Saúde

E-mail do autor principal: bruno.romano@odontologia.ufjf.br

\section{INTRODUÇÃO}

Reconstruções cirúrgicas na região bucomaxilofacial são extremamente desafiadoras pois a face é a área do corpo humano na qual uma assimetria por menor que seja já compromete a estética, além de gerar também distúrbios mastigatórios, desordens articulares e traumas psicossociais. Em casos complexos de anomalias craniofaciais, o bom planejamento cirúrgico é primordial para gerar resultados que corrijam as consequências dessas anomalias e evitem possíveis reincidências.

O modelo de planejamento cirúrgico tradicionalmente utilizado é constituído por muitas etapas laboratoriais, como análise de radiografias bidimensionais e simulação cirúrgica manual em modelo de gesso, as quais demandam muito tempo e por vezes podem levar a erros na cirurgia, dependendo amplamente da experiência clínica do cirurgião. O principal problema encontrado nesse tipo de planejamento é a falta de informações sobre a configuração tridimensional das estruturas relevantes envolvidas, como a linha média facial esquelética ou a articulação temporomandibular (HEUFELDER et al., 2017).

Recentemente com o desenvolvimento de softwares aplicados a cirurgias e o surgimento da impressão tridimensional, as novas técnicas de planejamento cirúrgico baseiam-se em modelos computadorizados do crânio do paciente, representando com precisão o crânio, a dentição, e o tecido mole facial (JI et al., 2019). Esses modelos de orientação após impressos facilitaram a transferência precisa do planejamento cirúrgico virtual para cirurgias reais realizadas em pacientes (LI et al., 2016; SEMBRONIO et al., 2020).

Nesse novo tipo de planejamento inicialmente os pacientes são submetidos a Tomografia computadorizada de Feixe Cônico (TCFC) para obtenção de imagens do crânio. Posteriormente, é realizado um escaneamento intra-oral desses pacientes para digitalização das arcadas dentárias, confeccionando ao final o modelo crânio-composto. Após isso, as análises

\section{E - book Tripé do Ensino Superior: Ensino,} Pesquisa e Extensão 
cefalométricas e simulações tridimensionais são feitas. Os guias cirúrgicos e placas de fixação são confeccionadas baseadas nos movimentos realizados através do planejamento virtual. Então a cirurgia é realizada a partir desse planejamento prévio (MAZZONI et al., 2015; SEMBRONIO et al., 2020).

Desse modo, o planejamento virtual auxilia na previsão dos resultados de uma intervenção cirúrgica antes da cirurgia real, permitindo aos cirurgiões que experimentem vários procedimentos de forma virtual. No entanto, mesmo com a previsibilidade ofertada pelo planejamento virtual, a qualidade do resultado cirúrgico depende diretamente da habilidade do cirurgião (MAZZONI et al., 2015).

Tendo em vista as mudanças geradas no planejamento cirúrgico com as novas tecnologias, o objetivo desta revisão é analisar as vantagens e indicações do planejamento cirúrgico virtual, destacando as inovações geradas por esse advento na prática cirúrgica.

\section{MÉTODO}

Esta é uma revisão integrativa que agrupa estudos que descrevem o uso do planejamento cirúrgico virtual para casos cirúrgicos de anomalias craniofaciais. O objetivo desta revisão é analisar nos estudos selecionados as vantagens e indicações do planejamento cirúrgico virtual, destacando as inovações geradas por esse advento para a prática cirúrgica.

Foram incluídos estudos observacionais (transversais, coorte, caso-controle e série de casos $\geq 5$ pacientes ou ensaios clínicos randomizados), revisões sistemáticas ou revisões integrativas. Além disso, foram priorizados estudos publicados entre 01 de janeiro de 2015 e 30 de dezembro de 2021, e redigidos em inglês ou português.

A busca foi realizada em duas bases de dados eletrônicas: Medline (via PubMed) e BVS, usando as estratégias descritas na Tabela 1. Foi usado filtro contemplando o período de publicação e os idiomas priorizados como critério de inclusão.

Tabela 1. Estratégia de busca nas bases de dados

\begin{tabular}{|c|c|}
\hline Base de dados eletrônica & Estratégia de Busca \\
\hline MEDLINE via pesquisa & (Orthognathic Surgery) And (Facial Asymmetry) \\
\hline PubMed estratégia: & And (Virtual Surgical Planning) And (Surgical Planning) \\
\hline Biblioteca Virtual em & (Orthognathic Surgery) AND (Facial Asymmetry) \\
Saúde (BVS) & AND (Virtual Surgical Planning) AND (Surgical Planning.)
\end{tabular}


Na primeira etapa da análise de dados a busca realizada na base de dados PubMed gerou 31 resultados, dos quais 8 foram excluídos a partir da análise dos títulos e resumos. Já na base de dados BVS foram gerados 33 resultados, dos quais 27 resultados eram duplicatas e foram excluídos e em seguida 5 foram excluídos a partir da análise dos títulos e resumos. Na segunda etapa, os 24 artigos selecionados foram lidos e analisados por completo, e desses, 5 foram excluídos por tratar de relatos de casos com $\leq 5$ pacientes e 1 foi excluído por fugir ao objetivo do presente estudo. Por fim, foram incluídos no estudo um total de 18 artigos.

Tabela 2. Resumo da Análise de Dados

\begin{tabular}{|c|c|c|c|}
\hline & & $\begin{array}{l}\text { PUBMED } \\
\text { 31 RESULTADOS }\end{array}$ & $\begin{array}{c}\text { BVS } \\
33 \text { RESULTADOS }\end{array}$ \\
\hline \multirow{3}{*}{$\begin{array}{l}1^{\mathrm{a}} \\
\text { ETAPA }\end{array}$} & $\begin{array}{l}\text { EXCLUÍDOS POR TÍTULO E } \\
\text { RESUMO }\end{array}$ & 8 & 5 \\
\hline & DUPLICATAS EXCLUÍDAS & 0 & 27 \\
\hline & ARTIGOS INCLUÍDOS & 23 & 1 \\
\hline \multirow{3}{*}{$\begin{array}{l}2^{\mathrm{a}} \\
\text { ETAPA }\end{array}$} & $\begin{array}{c}\text { EXCLUÍDOS: RELATO DE CASO } \\
\leq 5 \text { PACIENTES }\end{array}$ & 5 & 0 \\
\hline & $\begin{array}{c}\text { EXCLUÍDO POR FUGIR AO } \\
\text { TEMA }\end{array}$ & 0 & 1 \\
\hline & ARTIGOS INCLUÍDOS & 18 & 0 \\
\hline
\end{tabular}

\section{RESULTADOS E DISCUSSÃO}

A partir da análise dos artigos selecionados, pode-se observar como resultado que o planejamento tridimensional apresenta inúmeras vantagens, como simulação de osteotomias virtualmente, confecção de guias cirúrgicos, melhor visualização das estruturas ósseas, o que garante um resultado mais satisfatório (BENGTSSON et al., 2017; HAN et al., 2018; HSU et al., 2020; MAZZONI et al., 2015; SCOLOZZI, 2015; SEMBRONIO et al., 2020; QIN et al., 2019;).

A estética facial é um assunto complexo, pois além de toda funcionalidade da face como a mastigação, os sentidos, as ações musculares, é principalmente através dela e que ocorre a comunicação e a expressão das emoções. Dessa maneira, a deformidade craniofacial pode gerar um enorme impacto na vida do paciente, pois o comprometimento estético e funcional reflete diretamente na vida psicossocial do paciente e, por isso, a procura por tratamento é urgente e necessária. Dentre essas deformidades pode-se destacar a má-oclusão classe II ou III, 
hiperplasia ou hipoplasia condilar, dentre outras assimetrias faciais. (BELUSIC GOBIC, M. et al., 2021; HEINZMANN, G. et al.; 2021; PERKOVIC, V. et al., 2021)

O equilíbrio da simetria facial é determinado pela posição, volume e formato dos tecidos moles e duros (QIN et al., 2019). Nesse aspecto, a cirurgia bucomaxilofacial busca restituir a forma e função, estabelecendo um perfil estético, corrigindo a oclusão e consequentemente melhorando a qualidade de vida desses pacientes. Uma compreensão abrangente do problema é o primeiro passo antes de realizar a reconstrução definitiva. Portanto o planejamento préoperatório pode incluir fatores geométricos e quantitativos, como avanço de maxila, desprojeção da mandíbula ou combinação de ambos, com simulação virtual, otimizando o resultado. A análise e o planejamento tridimensionais são ferramentas poderosas em cirurgia craniofacial e reconstrutiva. Os procedimentos incluem 1) análise, 2) planejamento, 3) cirurgia virtual, 4) impressões 3D de guias ou implantes e 5) verificação dos resultados reais em relação aos planejados. O planejamento tridimensional e a cirurgia virtual aumentam a eficiência, precisão, criatividade e reprodutibilidade em cirurgia bucomaxilofacial (PARK et al., 2020; STEINBACHER et al., 2015).

Diferentemente, o planejamento convencional da cirurgia ortognática, anterior ao advento do planejamento virtual, é realizado com base em exames radiográficos, análise cefalométrica e simulação de cirurgia em modelos odontológicos fundidos em gesso, montados em articulador semiajustável. Infelizmente, o planejamento do modelo é complexo, e erros podem ser introduzidos durante a transferência do arco facial e uso de articuladores semiajustáveis, e as medidas de movimento do gesso pode ser impreciso. Também, placas intermediárias e finais manuais não são precisas. Além disso, a inclinação oclusal e uma deformidade assimétrica do esqueleto ósseo são difíceis de diagnosticar corretamente, mesmo com a recriação precisa do plano oclusal (DE RIU et al., 2017; JI et al., 2019). Portanto, em casos acentuados complexos de assimetria a técnica tridimensional apresenta uma vantagem maior, já que podem antecipar os movimentos cirúrgicos baseados na necessidade, como a mobilização translacional e rotacional do complexo maxilomandibular em seis direções diferentes. (BENGTSSON et al. 2017; HSU et al., 2020).

De acordo com Liao et al. (2020), o planejamento cirúrgico virtual tridimensional é superior na melhora da assimetria da linha média facial em comparação a abordagem convencional, porém os resultados de satisfação são igualmente elevados com os dois métodos de planejamento cirúrgico. Neste estudo, pacientes com deformidade classe III esquelética foram submetidos a cirurgia ortognática para correção da assimetria, podendo esta ser de várias formas: assimetria esquelética, assimetria de tecidos moles, assimetria funcional ou uma

\section{E - book Tripé do Ensino Superior: Ensino,} Pesquisa e Extensão 
combinação. Sendo, de suma importância que um diagnóstico preciso da assimetria seja realizado no pré-operatório para um planejamento e execução cirúrgica assertivos. A assimetria mais prevalente envolve desvios da mandíbula e da face. Destarte, o planejamento virtual tridimensional da cirurgia ortognática foi proposto para encurtar o tempo de planejamento, melhorar o planejamento cirúrgico, e aumentar a precisão cirúrgica em comparação com o planejamento convencional bidimensional. Embora a precisão cirúrgica seja um componente crítico do planejamento cirúrgico virtual, a avaliação das variáveis de resultado objetivas e subjetivas, bem como o tempo de avaliação, também é importante.

Atualmente os cirurgiões vêm adotando a abordagem CAD / CAM, que representa a união de duas tecnologias, CAD - desenho assistido por computador e CAM - manufatura assistida por computador. A abordagem CAD / CAM oferece diversas vantagens, incluindo: a visualização do local ideal para a osteotomia da maxila para restaurar uma relação ânteroposterior adequada, evitando lesões nos botões dos dentes permanentes, bem como potenciais interferências ósseas; manipulação de distratores virtuais, que permitem escolhas sobre o tamanho do distrator ideal e o posicionamento espacial tridimensional perfeito; transferência precisa do planejamento cirúrgico tridimensional para o paciente na sala de cirurgia por meio da fabricação de guias de corte cirúrgico específico e placas oclusais que mantêm o vetor do distrator; flexão das placas dos pés do distrator específico do paciente em um modelo craniano impresso em 3D feito por um processo de prototipagem rápida; eliminação da necessidade de manipulações intraoperatórias, sempre necessárias para o ajuste adequado dos distratores dobrados não customizados, reduzindo o tempo operatório para aproximadamente 45 a 60 minutos, que representa o tempo gasto para o planejamento da sessão virtualmente (SCOLOZZI, 2015).

A aplicação de placas cirúrgicas CAD/CAM específicas do paciente, guias de corte e implantes permitem um resultado satisfatório em cirurgia ortognática (SCOLOZZI, 2015), pois é possível maior controle vertical da maxila, o que implica numa melhor oclusão e posicionamento da mandíbula, entre outros segmentos ósseos (MAZZONI et al., 2015).

O fluxo de trabalho digital é essencial para que as limitações na precisão dos movimentos sejam superadas, visto que a simulação cirúrgica auxiliada por computador frequentemente combinada com uma etapa cirúrgica tradicional resulta em uma abordagem híbrida que expõe o resultado cirúrgico a uma possível margem de erro devido a imprecisões que ocorrem quando o plano digital é transferido na sala de cirurgia. $\mathrm{O}$ fluxo de trabalho digital conta com três fases sólidas: 1) simulação auxiliada por computador de cada procedimento cirúrgico; 2) projeto auxiliado por computador de ferramentas que permitem a transferência da 
cirurgia digital para o cenário real, como guias de corte cirúrgico e placas de osteossíntese ajustadas personalizadas; e 3) cirurgia sob a orientação de guias de corte impressos em 3D e placas personalizadas (MAZZONI et al., 2015; SEMBRONIO et al., 2020). Nesse âmbito, o planejamento virtual torna-se uma ótima escolha na resolução da maioria dos casos de deformidade craniofacial, não somente no planejamento cirúrgico, mas também em outras etapas essenciais do tratamento dentário.

De acordo com os estudos analisados, o planejamento virtual torna-se uma ótima escolha na resolução da maioria dos casos de deformidade craniofacial, não somente no planejamento cirúrgico, mas também em outras etapas essenciais do tratamento dentário.

Conforme Cintra et al. (2018), o planejamento virtual é utilizado também para o diagnóstico da assimetria facial e posterior tratamento ortodôntico, garantindo uma maior precisão ao longo de todo tratamento e maior previsibilidade. A partir do planejamento virtual tridimensional é feita uma análise das possibilidades mais indicadas para o preparo ortodôntico adequado em cada caso, e além disso, auxilia na escolha do melhor protocolo cirúrgico.

Bem como, segundo Ji et al. (2019), bons resultados são obtidos a partir do planejamento virtual com guias de osteotomia e placas de titânio pré-dobradas, visto que após a sobreposição da simulação cirúrgica e das imagens pós-operatórias, o posicionamento da maxila e mandíbula se mostraram satisfatórios, com maior acurácia para a maxila, com uma diferença linear média inferior a $0,60 \mathrm{~mm}$.

Além disso, técnicas que antes eram realizadas separadas, a partir do planejamento virtual podem ser realizadas no mesmo dia. Um exemplo é a cirurgia ortognática com condilectomia simultânea para o tratamento da hiperplasia hemimandibular/condilar. Os resultados cirúrgicos podem ser validados durante a cirurgia por navegação virtual. No entanto, o movimento de cada segmento ósseo não pode ser controlado com precisão conforme planejado antes da cirurgia (HAN et al., 2018; SEMBRONIO et al., 2020). Contudo, é importante salientar que na hiperplasia condilar, deve haver uma avaliação para se constatar se a doença está ativa ou inativa, em caso de inatividade a assimetria pode ser corrigida conforme os princípios ortognáticos (HIGGINSON et al., 2018).

Em relação a pacientes com microssomia craniofacial, estes apresentam uma ampla faixa de gravidade fenotípica. Existem vários sistemas de classificação clínica para características faciais de microssomias. As avaliações e intervenções devem ser sincronizadas com o crescimento craniofacial e adaptadas ao indivíduo com base nas características clínicas e nas preferências do paciente. As necessidades complexas dos pacientes são mais bem atendidas por uma equipe craniofacial multidisciplinar (BIRGFELD e HEIKE, 2019). Nesses

\section{E - book Tripé do Ensino Superior: Ensino,} Pesquisa e Extensão 
casos, o planejamento virtual é abrangente e fundamental. A oclusão final pode ser definida virtualmente ou com um registro de mordida. Além disso, os guias de corte necessários no lado afetado e uma orientação mesial permitirá um acesso mais fácil (POLLEY et al., 2019).

Contudo, a principal deficiência relacionada ao planejamento cirúrgico virtual, é o alto custo associado à técnica CAD / CAM. Outro potencial fator limitante poderia ser os erros cumulativos relacionados ao processo de aquisição de imagem e ao procedimento de software de aquisição de segmentação tridimensional que é transmitido ao fabricante o qual, de acordo com a literatura, foi estimado em $1 \mathrm{~mm}$ (SCOLOZZI, 2015). Outrossim, a simulação 3D dos tecidos moles também apresenta falhas pois ela ainda não permite o manejo preciso da altura facial e da posição do queixo. Diante esses fatos, faz-se necessário o monitoramento constante dos movimentos maxilares no transcirúrgico e também alguma margem para possível correção de imprecisões (DE RIU et al., 2017). Bem como, o aumento da exposição à radiação, uma vez que as Tomografias Computadorizadas de Feixe Cônico possuem uma exposição à radiação maior em comparação com a radiologia de projeção (LI et al., 2016).

\section{CONCLUSÃO}

O planejamento tridimensional representa uma inovação em cirurgia bucomaxilofacial. Este apresenta vantagens sobre o planejamento bidimensional, pois permite ao cirurgião realizar a avaliação do paciente com informações e imagens de estruturas craniofaciais livres de distorção de perspectiva, o que garante um melhor tratamento de deformidades, principalmente nas assimetrias unilaterais. No planejamento do tratamento há reduções de etapas e a utilização da técnica CAD / CAM permite uma maior precisão e previsibilidade, obtendo assim, resultados mais satisfatórios, visto que é possível realizar a simulação virtual das osteotomias virtualmente e confeccionar guias que auxiliarão durante a cirurgia.

\section{REFERÊNCIAS}

BELUSIC GOBIC, M. et al. Dentofacial deformity and orthognatic surgery: Influence on self-esteem and aspects of quality of life. J Craniomaxillofac Surg. v. 49, n. 4, p. 277-281 abr. 2021. DOI:10.1016/j.jcms.2021.01.024. Disponível em: https://pubmed.ncbi.nlm.nih.gov/33579617/. Acesso em: 13 set. 2021.

BENGTSSON, M. et al. Treatment outcome in orthognathic surgery-A prospective randomized blinded case-controlled comparison of planning accuracy in computer-assisted two- and three-dimensional planning techniques (part II). Journal of Cranio-Maxillofacial Surgery, [S. l.], v. 45, n. 9, p. 1419-1424, jul. 2017. DOI: 10.1016/j.jcms.2017.07.001. Disponível em: https://pubmed.ncbi.nlm.nih.gov/28800842/. Acesso em: 13 set. 2021. 
BIRGFELD, C. e HEIKE, C. Craniofacial Microsomia. Clinics in Plastic Surgery, [S. l.], v. 46, n. 2, p. 207-221, abr. 2019. DOI: 10.1016/j.cps.2018.12.001. Disponível em: https://pubmed.ncbi.nlm.nih.gov/30851752/. Acesso em: 13 set. 2021.

CINTRA, O. et al. Digital platform for planning facial asymmetry orthodontic-surgical treatment preparation. Dental Press Journal of Orthodontics, [S. l.], v. 23, n. 3, p. 80-93, maio/jun. 2018. DOI: 10.1590/2177-6709.23.3.080-093.sar. Disponível em: https://pubmed.ncbi.nlm.nih.gov/30088569/. Acesso em: 13 set. 2021.

DE RIU, Giacomo et al. Accuracy of computer-assisted orthognathic surgery. Journal of Cranio-Maxillofacial Surgery, [S. l.], v. 46, n. 2, p. 293-298, fev. 2018. DOI: 10.1016/j.jcms.2017.11.023. Disponível em: https://pubmed.ncbi.nlm.nih.gov/29275075/. Acesso em: 13 set. 2021.

HAN, B. et al. Hemimandibular Hyperplasia Correction by Simultaneous Orthognathic Surgery and Condylectomy Under Digital Guidance. Journal of Oral and Maxillofacial Surgery, [S. l.], v. 76, n. 7, p. 1563.e1-1563.e18, jul. 2018. DOI: 10.1016/j.joms.2018.03.006. Disponível em: https://pubmed.ncbi.nlm.nih.gov/29626447/. Acesso em: 13 set. 2021.

HEINZMANN, G. et al. Quality of life of patients following orthognathic surgery. Gen Dent. v. 69, n. 4, p. 64-69, jul.-ago. 2021. PMID: 34185671. Disponível em: https://pubmed.ncbi.nlm.nih.gov/34185671/. Acesso em: 13 set. 2021.

HEUFELDER, M. et al. Clinical accuracy of waferless maxillary positioning using customized surgical guides and patient specific osteosynthesis in bimaxillary orthognathic surgery. Journal of Cranio-Maxillo-Facial Surgery: Official Publication of the European Association for Cranio-Maxillo-Facial Surgery, [S. l.], v. 45, n. 9, p. 1578-1585, set. 2017. DOI: 10.1016/j.jcms.2017.06.027. Disponível em: https://pubmed.ncbi.nlm.nih.gov/28793965/. Acesso em: 13 set. 2021.

HIGGINSON, J.A. et al. Condylar hyperplasia: current thinking. British Journal of Oral and Maxillofacial Surgery, [S. l.], v. 56, n. 8, p. 655-662, out. 2018. DOI:

10.1016/j.bjoms.2018.07.017. Disponível em: https://pubmed.ncbi.nlm.nih.gov/30115459/. Acesso em: 13 set. 2021.

HSU, P. et al. Outcome of facial contour asymmetry after conventional two-dimensional versus computer-assisted three-dimensional planning in cleft orthognathic surgery. Scientific Reports, [S. l.], v. 10, n. 1, fev. 2020. DOI: 10.1038/s41598-020-58682-4. Disponível em: https://pubmed.ncbi.nlm.nih.gov/32047228/. Acesso em: 13 set. 2021.

JI, H. et al. Computer-assisted osteotomy guides and pre-bent titanium plates improve the planning for correction of facial asymmetry. International Journal of Oral and Maxillofacial Surgery, [S. l.], v. 48, n. 8, p. 1043-1050, ago. 2019. DOI: 10.1016/j.ijom.2019.01.026. Disponível em: https://pubmed.ncbi.nlm.nih.gov/30773336/. Acesso em: 13 set. 2021.

LI, Y. et al. Treatment of Dentofacial Deformities Secondary to Osteochondroma of the Mandibular Condyle Using Virtual Surgical Planning and 3-Dimensional Printed Surgical Templates. Journal of Oral and Maxillofacial Surgery, [S. l.], v. 74, n. 2, p. 349-368, fev. 
2016. DOI: 10.1016/j.joms.2015.06.169. Disponível em:

https://pubmed.ncbi.nlm.nih.gov/26183010/. Acesso em: 13 set. 2021.

LIAO, Y. et al. Outcomes of conventional versus virtual surgical planning of orthognathic surgery using surgery-first approach for class III asymmetry. Clinical Oral Investigations, [S. l.], v. 24, n. 4, p. 1509-1516, fev. 2020. DOI: 10.1007/s00784-020-03241-4. Disponível em: https://pubmed.ncbi.nlm.nih.gov/32100114/. Acesso em: 13 set. 2021.

MAZZONI, S. et al. Computer-Aided Design and Computer-Aided Manufacturing Cutting Guides and Customized Titanium Plates Are Useful in Upper Maxilla Waferless Repositioning. Journal of Oral and Maxillofacial Surgery, [S. l.], v. 73, n. 4, p. 701-707, abr. 2015. DOI: 10.1016/j.joms.2014.10.028. Disponível em:

https://pubmed.ncbi.nlm.nih.gov/25622881/. Acesso em: 13 set. 2021.

PARK, K. E. et al. Orthognathic Surgery to Improve Facial Profile: Assessment, 3-

Dimensional Planning, and Technique. Aesthetic Surgery Journal Open Forum, [S. l.], v. 3, n. 1, nov. 2020. DOI: 10.1093/asjof/ojaa051. Disponível em: https://pubmed.ncbi.nlm.nih.gov/33791672/. Acesso em: 13 set. 2021.

PERKOVIC, V. et al. Facial aesthetic concern is a powerful predictor of patients' decision to accept orthognathic surgery. Orthod Craniofac. [publicado online antes da impressão: 3 jun. 2021]. DOI:10.1111/ocr.12505. Disponível em: https://pubmed.ncbi.nlm.nih.gov/34080282/. Acesso em 13 set. 2021.

POLLEY, J. W. et al. Salvage or Solution: Alloplastic Reconstruction in Hemifacial Microsomia. The Cleft Palate-Craniofacial Journal, [S. l.], v. 56, n. 7, p. 896-901, dez. 2018. DOI: $10.1177 / 1055665618817669$. Disponível em:

https://pubmed.ncbi.nlm.nih.gov/30543122/. Acesso em: 13 set. 2021.

QIN, Z. et al. One-Stage treatment for maxillofacial asymmetry with orthognathic and contouring surgery using virtual surgical planning and 3D-printed surgical templates. Journal of Plastic, Reconstructive \& Aesthetic Surgery, [S. l.], v. 72, n. 1, p. 97-106, jan. 2019. DOI: 10.1016/j.bjps.2018.08.015. Disponível em:

https://pubmed.ncbi.nlm.nih.gov/30228074/. Acesso em: 13 set. 2021.

SCOLOZZI, P. Computer-aided design and computer-aided modeling (CAD/CAM) generated surgical splints, cutting guides and custom-made implants: Which indications in orthognathic surgery? Revue de Stomatologie, de Chirurgie Maxillo-faciale et de Chirurgie Orale, [S. l.], v. 116, n. 6, p. 343-349, dez. 2015. DOI: 10.1016/j.revsto.2015.09.005. Disponível em: https://pubmed.ncbi.nlm.nih.gov/26598243/. Acesso em: 13 set. 2021.

SEMBRONIO, S. et al. One-Stage Computer-Guided Customized Management of Skeletal Asymmetry by Concomitant Proportional Condylectomy and Orthognathic Surgery in Patients With Unilateral Condylar Hyperplasia. Journal of Oral and Maxillofacial Surgery, [S. l.], v. 78, n. 11, p. 2072.e1-2072.e12, nov. 2020. DOI: 10.1016/j.joms.2020.05.039. Disponível em: https://pubmed.ncbi.nlm.nih.gov/32621806/. Acesso em: 13 set. 2021.

STEINBACHER, D. M. Three-Dimensional Analysis and Surgical Planning in Craniomaxillofacial Surgery. Journal of Oral and Maxillofacial Surgery, [S. l.], v. 73, n. 12, p. S40-S56, dez. 2015. DOI: 10.1016/j.joms.2015.04.038. Disponível em: https://pubmed.ncbi.nlm.nih.gov/26608154/. Acesso em: 13 set. 2021. 